\title{
Towards the decentralized revolution in energy systems using blockchain technology
}

\author{
Umit Cali ${ }^{*}$, Austin Fifield \\ Engineering Technology and Construction Management, University of North Carolina at Charlotte, 9201 University City Blvd, \\ Charlotte 28223, USA
}

\begin{abstract}
Micro and decentralized generation of electrical power is an emerging trend in the power industry. Blockchain technology allows decentralized and instant monetary and contractual transactions to occur over peer to peer private and public networks by satisfying cyber security concerns. Both changes have the potential to enable paradigm shift in the field of energy in the coming years. Decentralization in the energy business makes this sector a good playground for blockchain technology. As of now, energy applications of blockchain technology are in conceptual and early stage prototyping levels. This paper aims to present a comprehensive review of P2P energy trading related blockchain technology applications and proposes a comprehensive multi-layer energy model architecture for the peer to peer (P2P) energy trading implementations where the use of blockchain technology is integrated. P2P energy trading allows consumers to become prosumers of electricity in a more efficient, trustable, and profitable way. Furthermore, this study demonstrates an Ethereum based blockchain testbed exhibiting blockchain concepts and how they can be used in the field of P2P energy trading within a commodity microgrid using a sample use-case scenario.
\end{abstract}

Keywords: Microgrids, Blockchain Technology, Peer-to-Peer Energy Trading, Multi-Layer Energy Model Architecture

\section{Introduction}

Historically, centralized energy generation has been used to satisfy the electricity demand. The majority of the centralized energy generation assets use non-renewable primary energy resources, such as coal and natural gas. After the global oil crises in the 1970s, most governments started to run pushpolicies to increase the portion of domestic energy resources by supporting renewable and decentralized energy resources, such as wind and solar energy. Meanwhile, the liberalization process of power markets had been completed in most countries. Market players and the power market responded with pushpolicies which were also designed to subsidize new and renewable energy resources in the liberal energy markets. The manufacturers of renewable energy system components started to lower the costs of higher efficient systems which increased the economic competitiveness of renewable energy resources (RES). Decentralized energy generation reduces the overall energy losses and decreases the carbon-dioxide emissions due to less physical distances between energy supply and demand sites [1]. The majority of the decentralized energy resources are in the form of RES which are intermittent and make it difficult to predict their power output [2] [3]. High intermittency of RES causes additional uncertainty in the supplydemand balance and increases the balancing power costs. At present, the vast majority of power markets are using merit-order principle to determine the electricity prices on a national or large-regional level which does not reflect the micro-level and local energy balance conditions. Current wholesale markets are not able to mimic in [near] real-time to the intermittent power generation characteristics of RES [4]. Since

\footnotetext{
* Manuscript received April 25, 2018; revised March 16, 2019.

Corresponding author. Tel.: 001+9802593583; E-mail address: ucali@uncc.edu.

doi: 10.12720 /sgce.8.3.245-256
} 
future energy markets tend to evolve into more decentralized markets, development of new local market design efforts become more critical to operate decentralized power markets. Et al. [5] presented new local market approaches which support the successful integration of distributed RES into the power grid and markets without the use of secure power transactions using blockchain technology. The improvements in renewable energy power prediction tools, almost real-time controlling of decentralized power generation assets using advanced control algorithms, and finally new generation information communication technologies (ICT), accelerate the transformation rate of the future energy markets. Almost real-time monitoring of energy supply-demand nodes and monetary transaction are two key elements of future energy markets. Besides power markets, other sectors are evolving into the decentralized economy. Blockchain technology is an emerging technology which will transform the future business and social structures.

Both decentralized energy generation and blockchain technology are enabling more democratic and decentralized markets (see Fig. 1). Future power networks will host more decentralized and new components with increased autonomy. The increased use of electric vehicles is expected to increase the complexity of future power systems and markets.

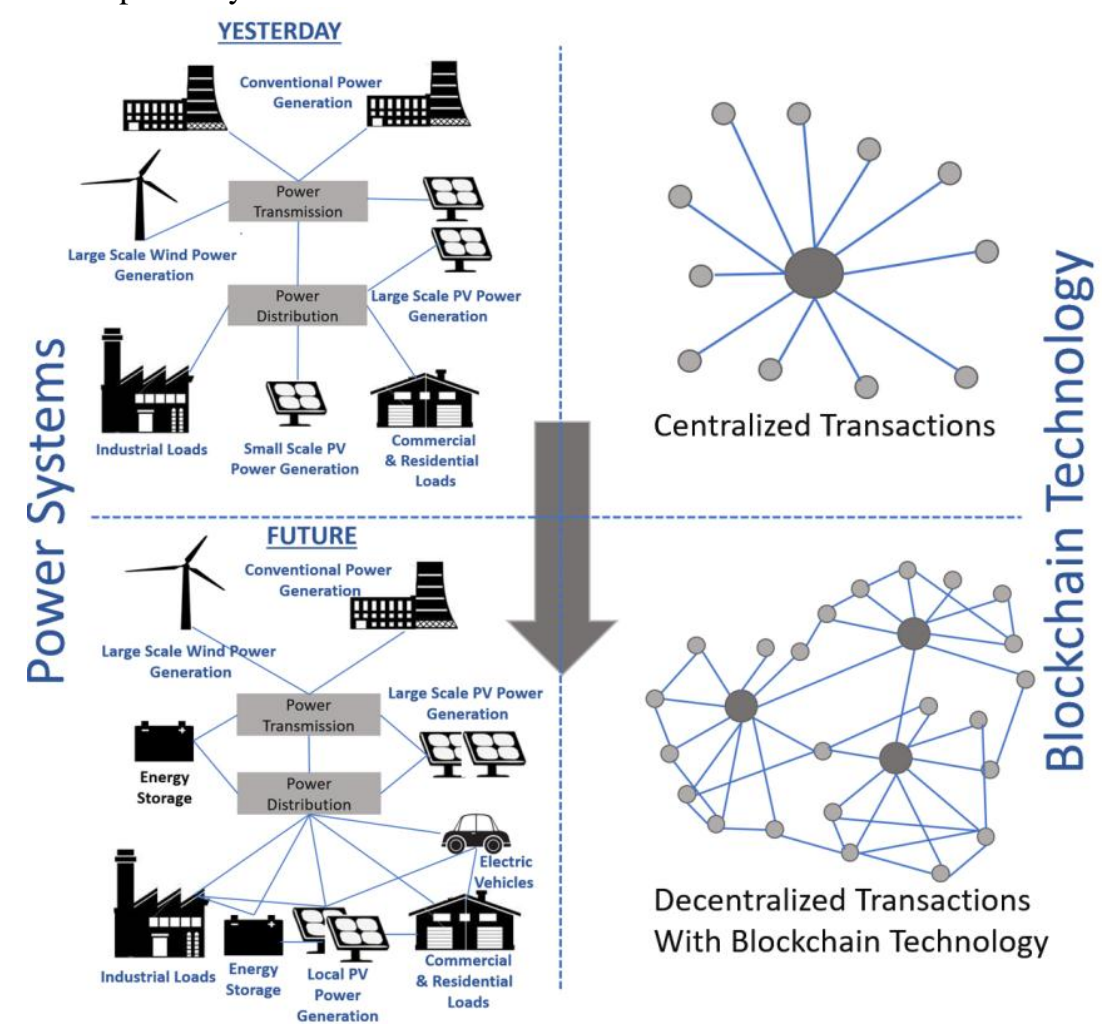

Fig. 1. Decentralization of power systems and blockchain technology.

Blockchain technology is a perfect match for future power markets and grids which empowers the decentralization at the core. Blockchain technology allows decentralized and instant monetary and contractual transactions over the peer to peer private and public networks by satisfying the cyber security concerns. Blockchain technology can also be defined as a distributed database. Blockchain technology can be implemented in the energy field in several potential areas such as smart-meter monitoring and billing, dynamic-demand-side management, clean energy development (CDM) projects, carbon-dioxide trading, renewable energy certificate (REC) trading, mezzanine financial applications of energy efficiency projects, electric vehicle charging applications and $\mathrm{P} 2 \mathrm{P}$ energy trading. This study is focused on the P2P energy trading implementations of blockchain technology. 
P2P energy trading supports the smooth operation of the power grid especially on power systems where the penetration level of intermittent power resources such as wind and solar are high. Future power system structures will be more decentralized with new players such as prosumers. Real-time power trading between prosumer peers and other decentralized power market players will require an accurate track-record of the power and corresponding monetary transactions in a thrustless environment by satisfying cyber security concerns [6].

This article aims to present a comprehensive review of P2P energy trading related blockchain technology applications and proposes an extended multi-layer energy model architecture for smart energy applications such as peer to peer ( $\mathrm{P} 2 \mathrm{P})$ energy trading, where the use of blockchain technology is integrated alongside other cyber-physical components. The conceptual studies are essential for proper road-mapping and standardization of emerging technologies and their implementations on future power systems. This is the first paper (to our knowledge) to explore the implementation of blockchain technology on the multi-layer energy model architecture. Furthermore, this study demonstrates an Ethereum based blockchain testbed exhibiting blockchain concepts and how they can be used in the field of P2P energy trading within a commodity microgrid using a sample use-case scenario.

\section{Literature Review}

\subsection{Decentralized power and microgrid markets}

Decentralized power generation of electrical power typically consists of small, medium, or low-scale voltage connected units. Most of the photovoltaic solar power capacity is installed close to the energy consumption side. Microgrids are decentralized systems which may be operated on or off-the-grid depending on the conditions [7]. Microgrids give the opportunity to trade local generated electrical power on the local or regional power markets. Most of the current peer to peer (P2P) energy trading models are operated geographically independent where generation and demand-side units are located in different locations. The virtual power plant concept is used to enable the use of regional microgrid transactions. Participants of the virtual microgrid system are considered as virtual communities. Community microgrids are designed to feed the local communities' electrical or heat demands by using their own energy generation assets and enabling new opportunities for the shared economy. Community based microgrids use similar ICT infrastructure like virtual power plants where most of the power system components are connected physically on-site. This concept has the potential to reduce grid transmission and distribution losses and discard some third-party companies between the power generator and consumers. Therefore, community microgrids can reduce the carbon-dioxide emissions by decreasing power system losses. Besides, during power black-out times after man-made or natural disasters, community microgrids are capable of increasing the resiliency of the local community. Recently, energy companies and utilities have initiated a couple of microgrid pilot projects. The following companies are currently in operation in the P2P energy trading business:

- Energy Internet, China

- PeerEnergyCloud, Germany

- Smart Watts, Germany

- Sonnencommunity, Germany

- Lichtblick Swarm Energy, Germany

- Community First! Village, US

- Electron, UK

- Cornwall Energy,UK

- Vandebron, Netherlands

- Piclo, UK

- Sonnen GmbH, Germany

- Power Ledger, Australia

- PowerPeers, Netherlands 
Piclo was established in 2014 in the UK as well as Open Utility (technology company) and Good Energy (distributed energy supplier). As of now, Piclo is collaborating with ERG and Essent alongside Good Energy. Piclo's developed business model enables P2P transactions between consumers and local renewable energy providers. Their model is operational at a national level [8]. SonnenCommunity in Germany: Sonnen GmbH has started operations in 2015 in Germany and extended their operations to other countries such as the US, Netherlands, and Australia. SonnenCommunity claims to own the largest $\mathrm{P} 2 \mathrm{P}$ platform in the world. The company provides $\mathrm{P} 2 \mathrm{P}$ trading capabilities using energy. Power Ledger Ecosystem supports various energy trading applications including P2P energy trading and wholesale and distributed markets. Power Ledger developed a dual token Ecosystem (POWR and Sparkz) which is functional on two blockchain layers. Sparkz tokens are priced, transacted, and redeemed in the local currency environments and POWR tokens are needed to produce Sparkz tokens [9]. PowerPeers is a startup P2P energy company which was launched by Vattenfall in the Netherlands. The company specializes in a digital interactive marketplace for P2P energy trading. LO3 launched TransActive Grid in Brooklyn, NY and initiated a pilot project delivering real-time metering of local energy generation in their community microgrid, naming it the Brooklyn Microgrid [10] and [11].

\subsection{Blockchain technology}

Blockchain Technology is an innovative technology designed to decentralize transactions while also creating additional security. In other words, blockchain technology is a type of distributed database (ledger) which can securely host critical information such as contracts, data, events and monetary transactions [12]. Critical data is stored in 'blocks' and linked with 'chains' to the preceding block using a cryptographic hash function.

Each ledger is validated and verified by a network of nodes (participants) according to a set of rules, called a consensus, within the virtual network. The virtual network can be private or public depending on the application and use-case. This structure does not require any centralized authority. This process produces a digital ledger containing data incapable of corruption. These transactions are verified and fulfilled with blocks generated on the blockchain before becoming a block of their own. This ongoing verification process produces a database of every transaction, verifiable by each node connected to the network. Nodes are able to connect to the network independently, updating themselves to match the shared data from all nodes on the network. All nodes on the virtual network hold a full copy of the entire preceding ledgers [13].

Blockchains utilize an embedded cryptocurrency for trading amongst nodes. Some blockchains are tokenized, allowing users to trade using their own private token currency. This is especially important when used in private blockchain networks for trading monetized physical entities, such as goods, services, energy, etc. For the blockchain to allow the trading of external services, it must receive data externally. This data cannot be received through one of the connected nodes as it cannot be verified and confirmed as factual. For this data to embed itself into the blockchain, the network must be slightly centralized through the use of an Application Program Interface (API) hosted through nodes on the network. Blockchain APIs can be used to develop software for the blockchain producing data deemed legal and factual by all nodes on the network. Though the blockchain becomes slightly centralized, all transactions between nodes on the network are verified and securely fulfilled. A blockchain also uses APIs for interfacing the network with human peers to easily and quickly allow transactions to occur.

Bitcoin uses a proof-of-work (PoW) consensus which was introduced by Satoshi Nakamoto. PoW creates a distributed thrustless consensus and solves the redundant-spend problem where very high computational power is required to maintain the system [14].

Ethereum is a powerful decentralized blockchain platform which runs smart contracts. Ethereum is capable of not only allowing the trade of their cryptocurrency but also contains a smart contracting feature. These contracts are typically programmed in a language called "Solidity". The blockchain uses a compiler to read this contract and embeds it into the blockchain. This contract sets rules and guidelines for specific transactions on the network. When all terms of the contract are met, the transaction will be 
fulfilled and verified by the blockchain. This dynamic, semi-centralized network has many applications. Its use in energy trading will be examined.

IOTA is a crypto-currency developed for the M2M and IoT platforms which is based on Tangle, a blockchain "without blocks". Therefore, IOTA can be considered as a ledger of things and works according to the distributed ledger principle. Zero fees and low power consumption features make IOTA more attractive for future energy applications. This technology is still under development and not fully tested [15] and [16].

The energy systems related to blockchain technology is a new field and only conducted by few researchers. The existing research focuses mainly on impacts, security, and scalability of the blockchain network using industrial internet of things, optimization, and demand response programs of the microgrids networks and energy grids. The decentralized optimization of energy resources in microgrid networks using blockchain technology is presented in [17]. Scalability, security and use of industrial IoT related research topics are investigated under the consortium blockchain concept for energy trading usecases in [18]. Demand response management, microgrids energy market design, and employment of virtual currency for trading the RES using blockchain technology are proposed in various literature [2], [19], [20] and [21].

The existing literature does not cover a comprehensive top-bottom approach which may help to identify energy related use-cases of blockchain technology in a systematic way by considering the high complexity of current and future energy systems. The proposed 7-layer smart grid - blockchain technology architecture model is designed to explore the big picture and important interactions within the complex interdisciplinary environment. Therefore, the model has potential to impact future research activities by guiding researchers in a more structured manner.

\section{Interactions between Power Systems and the Blockchain Technology}

A 5-Layer Smart Grid Architecture Model (SGAM) has been used as a reference model for several European Union R\&D projects to achieve a common understanding and standardization of smart grid modelling efforts. [22], [23], [24] and [25] proposed a 4-layer architecture model without considering the use of blockchain technology in their article. The previous models did not provide higher resolution which can be used to discuss additional extensions of the topic. Besides, there has been no other existing article detected where the employment of blockchain technology is discussed on such multi-later energy model structures. Therefore, this article proposes a 7-layer architecture energy model where the impacts and interactions of blockchain technology on possible energy related use-cases in a multi-domain complex structure are investigated. (see Fig. 2)

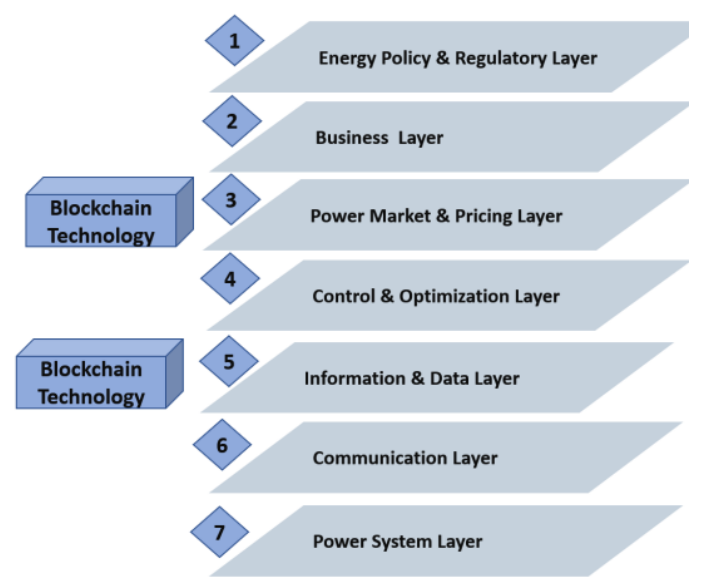

Fig. 2. 7-Layer architecture model of power systems and blockchain technology interactions. 


\subsection{Energy policy and regulatory layer}

The policy makers define the rules of the game. The energy policy and regulatory framework layer covers all monetary and technical issues. Policy makers are responsible for creating legislative documents to satisfy the country's or state's energy safety and security functions. Energy security is one of the primary responsibilities of most governments in the world. All players on the market are responsible for obeying the rules which are determined by the policy makers. Regulatory authorities are neutral entities which can observe and investigate market operations and stakeholders as well as check if they operate according to the legislative documents. They may report policy makers if there will be a need to adjust existing legislative documents due to energy laws not satisfying the effective operation of the market in the healthiest way. Updated legislative documents can then be proposed and executed by using a country's specific path before they will be officially published. There are some legislative and regulatory efforts needed to promote the use of blockchain technology which is expected to be one of the main issues for policy makers.

\subsection{Business layer}

Investors of energy assets, trading companies, and transmission and distribution system operators are stakeholders of the conventional power markets. In the recent decade, new players are included in the market such as prosumers and other innovative service provider companies. Secondary level stakeholders which may include service and product providers who support the sustainability of the power systems and markets such as manufacturers of power generation, transmission and distributions infrastructure, ICT providers and various project development and consultancy companies. Economic decisions are given by considering the energy policy and regulatory layer and the general economic metrics, which are used for any investment decision process, such as return on investment and discounted payback periods. The cost and benefits analysis of blockchain technology can be considered one of the new fields for the energy economists and investors. The cost of required energy, communication and computation hardware and software are some of the cost elements of the future sharing economy where blockchain technology will be used dominantly.

\subsection{Power markets and pricing layer}

Electricity and the power market is a complex system which enables monetary transactions between the buyer and seller of electrical power. Liberalization processes of the power markets have transformed market conditions and structures during the last two decades. Market rules of the competitive power markets allow independent power producers and non-utility power producers to trade electrical power. $\mathrm{P} 2 \mathrm{P}$ market place rules are still under development. [26], [27] and [28] researchers proposed various $\mathrm{P} 2 \mathrm{P}$ market mechanism structures. Block et al. [28], Ilic et al. [29] and Liu et al. [30] emphasized the rules of P2P decentralized power markets based on blockchain technology. Blockchain technology has already been used by several companies as discussed in detail in previous sections. The cryptocurrency feature of blockchain technology still has to be further improved for more advanced and user-friendly applications in the future.

\subsection{Control and optimization layer}

Supervisory Control and Data Acquisition (SCADA) systems have been standards for the power industry for more than two decades. SCADA, and similar advanced programs, are used to control the operation of intelligent energy systems to include the optimization and decision-making algorithms performed in this layer. It is expected that the data analytics such as the use of artificial intelligence (AI) and advanced decision-making processes will be integrated with blockchain technology in the coming years. 


\subsection{Information and data layer}

Data processing, analysis and cyber security functionalities are handled under the information and data layer. This layer is built on the communication layer. Digital contracting and consensus mechanisms are functioning under this layer. It is necessary to improve cyber security standards of various communication technologies which are used in parallel to the blockchain platforms.

\subsection{Communication layer}

The Communication Layer hosts various communication protocols which are responsible for interaction between different layers. The reliability, scalability, security, power consumption levels and economic viability of the communication technologies and protocols are critical to allow the efficient interoperability of power systems; especially smart-grids. The following communication protocols are used in energy systems:

- $\quad$ Standard internet protocols such as IPv4 and IPv6

- IEEE 1901 - Power Line Communication (PLC)

- $\quad$ IEEE 802.3 - Ethernet

- $\quad 3 \mathrm{GPP}$ - Long-term Evolution (LTE)

- $\quad$ IMT 2000 - Third generation wireless telecommunication technology $3 \mathrm{G}$

- $\quad$ IMT 2000 - Fourth generation of broadband cellular network technology 4G

- IEEE 802.15.3: High Rate Wireless Personal Area Network (WPAN)

- IEEE 802.11 - WIFI

- $\quad$ IEEE 802.15.4 - Zigbee

- IEEE 802.16x -Worldwide Interoperability for Microwave Access (WIMAX)

The communication and information infrastructure shall also be energy efficient and reliable to maintain the needs of the industry where blockchain technology is implemented.

\subsection{Power systems layer}

Physical components including power generation, transportation and distribution units on the generation side, and consumers or prosumers on the demand side, can be listed under the power systems layer.

\section{Use Case: Sample Peer to Peer Energy Trading Testbed}

\subsection{Methodology and limitations}

In modern energy markets, prosumers cannot be instantly rewarded for the energy they sell back to the grid. Instead, utility companies purchase excess energy produced by the prosumer as a monthly credit [31]. There are also many areas where prosumers cannot sell energy back to the grid as well as other areas where sell-back prices are only a small fraction of the price utility companies charge for grid-consumed energy. This so called "net metering" approach does not give the prosumer the price at which they would like to sell their energy, nor does it give them the ability to sell the energy to specific consumers. Incorporating blockchain into the trade of electricity will give the prosumer more freedom with the energy they have produced. The prosumer is also rewarded nearly instantly through secure transactions on the blockchain. However, there are some limitations to using blockchain technology in this application. When the chain develops, with tens of thousands or even millions of transactions, not only with the storage space of the connected devices be tested, but new nodes joining the network may have processors with speeds far too slow. There are also physical limitations to this system. The resolution of transactions, i.e. how often the prosumer gets rewarded for their produced energy, is extremely important in determining energy storage size and overall system costs. A fine resolution will reduce system costs as well as decrease prosumer reward wait times. This resolution is dictated by Smart Contract capabilities and device storage and processing speed limitations. 


\subsection{Components of the testbed}

The testbed consists of two Linux OS nodes running on separate computers, two mining nodes also running on Linux OS on separate computers, wireless IEEE 802.11 connection, stand-alone photovoltaic nodes, and related software to run the blockchain. All nodes each contain two independent accounts, creating a network of effectively eight nodes. These nodes act as virtual energy consumers on the network. The photovoltaic nodes produce energy and store them in sealed, deep cell batteries. These nodes act as prosumers on the network, having the ability to sell their stored energy to other nodes on the blockchain network.

\subsection{Development of private blockchain network}

A private blockchain test network has been designed and built for proof of concept and testing of potential energy trading concepts. The blockchain network utilizes Ethereum, an open source blockchain platform, capable of running smart contracts; allowing nodes to fulfil transactions securely when both parties agree to the terms defined in the contract [32]. Transactions are typically performed in Ether (Ethereum's cryptocurrency), but with tokenization, transactions can occur using virtual tokens within the private network.

The backbone of the blockchain is Go-Ethereum (Geth). Geth is a command-line based interface running Ethereum on each node, allowing the nodes to communicate securely. Geth is written in "Go", a programming language which allows the creation of reliable and efficient software [33]. Geth is installed on every node so that each can communicate securely. When the blockchain is created, the difficulty level for block creation is set. It is important to set an easy difficulty level when using a private network as these blocks are required to push transactions and must be produced as quickly and efficiently as possible. The miners are awarded with Ether however because the private network is tokenized, this Ether has no market value and is simply a biproduct of the private blockchain. To quickly and efficiently mine blocks for these transactions, the mining nodes are utilized for this purpose. These blocks are also used to verify each transaction. A maximum of twelve blocks are used to verify and confirm each transaction. Each transaction also costs "gas" to complete. The gas level for this blockchain is set extremely low when setting up the network. This allows for many transactions to occur without draining the nodes of Ether. Each node is set up with a large amount of private Ether to ensure all transactions can always occur, even if the smart contracts are created inefficiently, costing more gas than necessary.

The initial testbed assumes functionality under an unregulated market or possibly a new market containing power systems where P2P energy trading is supported. As observed in Fig. 3, along with the visualization of power and money flow, there are three sample nodes which represent separate households inside the community microgrid where the private blockchain rules are valid. Node 1 is the only prosumer node with installed PV generation capacity. Other nodes are functioning as consumers. Smart contracts have been created for this blockchain to allow the trading of Energy Tokens. These tokens have no real market value outside of the private blockchain. The tested, which is presented in this study, shows only the private blockchain scenario. They have an equivalent value inside the blockchain and are traded in return for excess energy produced by nodes with renewable energy sources. The contract between two nodes lists the terms and conditions of the transaction. When a node would like to sell its stored energy, it calls the contract from the blockchain, enters this energy value, and in return, receives an equivalent energy token from the nodes it traded with. These tokens can be purchased from the mining nodes with either physical USD, or a cryptocurrency outside of the private blockchain network. Though the network is decentralized with energy transactions, the mining nodes act as a "bank" for the purchasing of tokens. These token-purchasing transactions with the "bank" also occur with the use of a smart contract. Currently, a graphical user interface (GUI) called "Mist" is being utilized to fulfil these transactions and view energy token account balances. There are many other interfaces on the market and many blockchains should utilize their own GUI to interface with mobile phone applications with web-based access. 


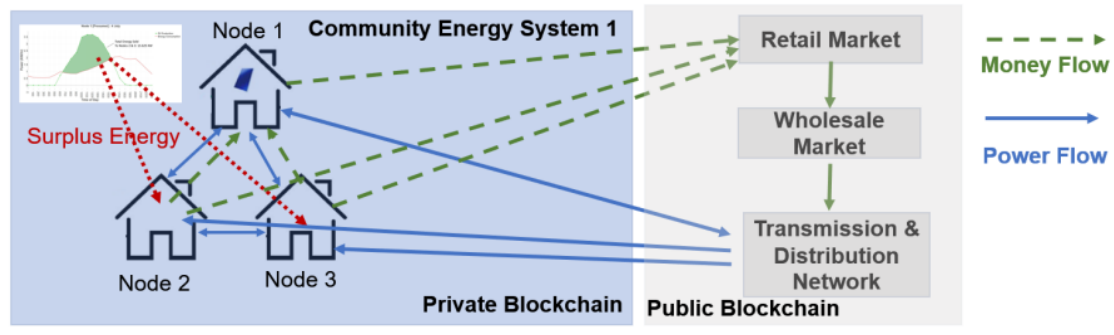

Fig. 3. Simple P2P Trading Architecture.

\subsection{Experimental results}

Utilizing the private blockchain, energy is traded amongst peers in exchange for tokens. Nodes are paid off by the blockchain in a real cryptocurrency at predetermined intervals to reward a real market currency for their energy. Below, Fig. 4, 5 and 6 illustrate the effectivity of blockchain technology in a peer to peer energy trading scenario. Fig. 4 demonstrates the prosumer's involvement in this network. Surplus energy produced during the day (typically during the hours of 7:00am - 3:00pm), is sold to consumer nodes on the blockchain. This data is consistent with a $5 \mathrm{~kW}$ photovoltaic system installed on a household in San Diego, California, USA with real energy consumption values. In this instance, this home generates $13.625 \mathrm{~kW}$ of surplus energy during 4 July. Equation (1) is used to calculate this value. Parameter descriptions of Equation (1) are given under Table 1.

Table 1. Parameter description for the Equation 1.

$S E=\sum_{t=0}^{t=n}[\mathrm{p}(t)-\mathrm{c}(t)] \quad$ Equation 1

\begin{tabular}{|l|l|}
\hline SE & Energy sold from the prosumer in kilowatts \\
\hline$p$ & Energy produced from the prosumer in kilowatts \\
\hline $\mathrm{c}$ & Energy consumed by the prosumer in kilowatts \\
\hline
\end{tabular}

Where $\mathrm{t}=0$ is the first instance at which the energy produced by the panel, minus the energy consumed by the household, is greater than 0 , and $\mathrm{t}=\mathrm{n}$ is the last instance when this condition exists.

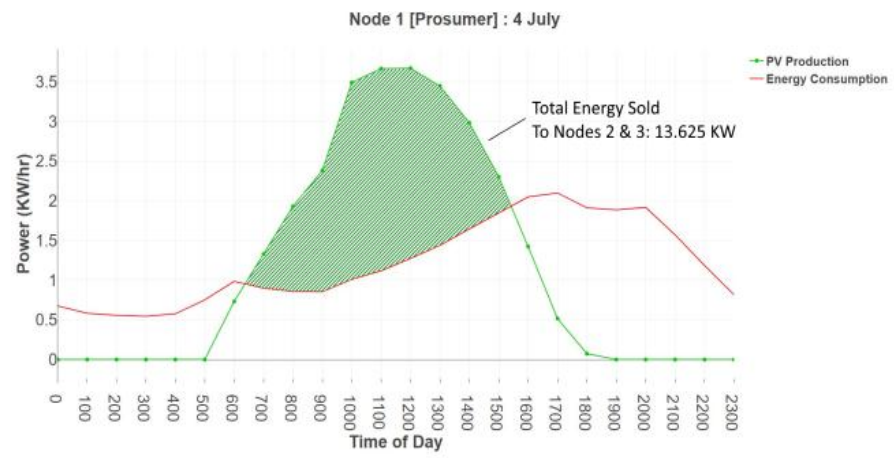

Fig. 4. Node 1 Energy production and consumption.

Fig. 5 illustrates Node 2; a consumer's impact on the blockchain. During the hours in which Node 1 had a surplus in energy, Node 2 purchased that energy and reduced it from its overall energy usage during that time of the day. When there are extreme peaks in energy surplus that surmount the energy required to reduce the consumption to zero, that excess energy is then sold to another consumer on the blockchain. Between the hours of 8:00am and approximately 1:00pm, the surplus energy produced from Node 1 exceeded the energy consumed by Node 2. This allowed Node 2 to purchased $8.957 \mathrm{~kW}$ from the available $13.625 \mathrm{~kW}$ from Node 1 . The remaining $4.668 \mathrm{~kW}$ is then sold to Node 3 during those hours (see Fig. 6). 


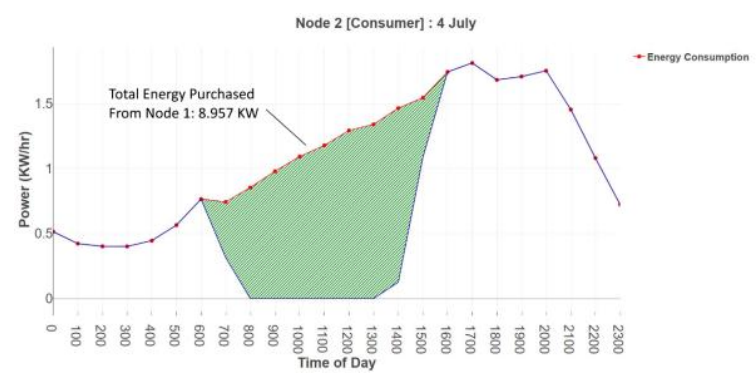

Fig. 5. Node 2 Energy consumption including purchased additional energy.

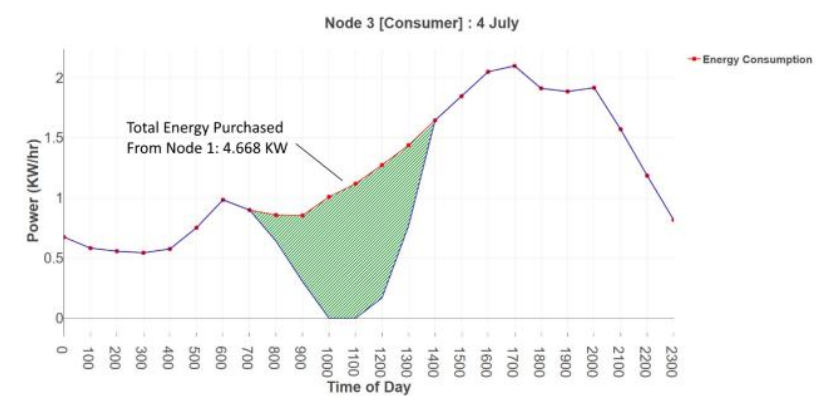

Fig. 6. Node 3 Energy consumption including purchased additional energy.

The energy purchased and sold over the blockchain is done with private blockchain tokens. A summary of the token results can be viewed in Table 2. Each node begins the day with an arbitrary number of tokens (100). These tokens would have been purchased ahead of time by the nodes with actual currency. For every 150 watts of energy, 1 token can be traded. This allows Node 1 to received 90.834 tokens by the end of the day from Nodes 2 and 3 collectively. Nodes 2 and 3 lose tokens directly proportional to the amount of energy they purchased during that day. This creates a quick and efficient system for securely trading energy between peers.

Table 2. Blockchain tokenization for simulated one day.

\begin{tabular}{|c|c|c|c|c|c|c|}
\hline Time of Day & \begin{tabular}{|c|} 
Node 1 \\
Energy \\
Surplus (KW) \\
\end{tabular} & $\begin{array}{l}\text { Node } 1 \\
\text { Energy } \\
\text { Tokens } \\
\end{array}$ & $\begin{array}{c}\text { Node } 2 \\
\text { Purchased } \\
\text { Energy }(\mathrm{kW}) \\
\end{array}$ & $\begin{array}{l}\text { Node 2 } \\
\text { Energy } \\
\text { Tokens }\end{array}$ & $\begin{array}{c}\text { Node } 3 \\
\text { Purchased } \\
\text { Energy }(\mathrm{kW}) \\
\end{array}$ & $\begin{array}{l}\text { Node } 3 \\
\text { Energy } \\
\text { Tokens }\end{array}$ \\
\hline $0: 00$ & 0 & 100 & 0 & 100 & 0 & 100 \\
\hline $1: 00$ & 0 & 100 & 0 & 100 & 0 & 100 \\
\hline $2: 00$ & 0 & 100 & 0 & 100 & 0 & 100 \\
\hline $3: 00$ & 0 & 100 & 0 & 100 & 0 & 100 \\
\hline $4: 00$ & 0 & 100 & 0 & 100 & 0 & 100 \\
\hline $5: 00$ & 0 & 100 & 0 & 100 & 0 & 100 \\
\hline $6: 00$ & 0 & 100 & 0 & 100 & 0 & 100 \\
\hline $7: 00$ & 0.427852 & 102.852 & 0.427852 & 97.148 & 0 & 100 \\
\hline $8: 00$ & 1.071612 & 109.996 & 0.854339 & 91.452 & 0.217273 & 98.552 \\
\hline 9:00 & 1.52778 & 120.182 & 0.978549 & 84.928 & 0.549231 & 94.89 \\
\hline $10: 00$ & 2.4826 & 134.188 & 1.092013 & 77.648 & 1.008922 & 88.164 \\
\hline $11: 00$ & 2.547426 & 149.495 & 1.177621 & 69.798 & 1.11851 & 80.707 \\
\hline $12: 00$ & 2.396835 & 165.475 & 1.292576 & 61.18 & 1.104259 & 73.345 \\
\hline $13: 00$ & 2.009288 & 178.87 & 1.339558 & 52.25 & 0.66973 & 68.88 \\
\hline $14: 00$ & 1.33733 & 187.786 & 1.33733 & 43.334 & 0 & 68.88 \\
\hline $15: 00$ & 0.457257 & 190.834 & 0.457257 & 40.286 & 0 & 68.88 \\
\hline $16: 00$ & 0 & 190.834 & 0 & 40.286 & 0 & 68.88 \\
\hline $17: 00$ & 0 & 190.834 & 0 & 40.286 & 0 & 68.88 \\
\hline $18: 00$ & 0 & 190.834 & 0 & 40.286 & 0 & 68.88 \\
\hline $19: 00$ & 0 & 190.834 & 0 & 40.286 & 0 & 68.88 \\
\hline $20: 00$ & 0 & 190.834 & 0 & 40.286 & 0 & 68.88 \\
\hline $21: 00$ & 0 & 190.834 & 0 & 40.286 & 0 & 68.88 \\
\hline $22: 00$ & 0 & 190.834 & 0 & 40.286 & 0 & 68.88 \\
\hline 23:00 & 0 & 190.834 & 0 & 40.286 & 0 & 68.88 \\
\hline
\end{tabular}




\section{Conclusion}

The energy industry, especially with peer-to-peer (P2P) energy trading applications, is a perfect implementation field for blockchain technology which has a great potential to change the business models, market conditions and human behavior in the future. In this article, a comprehensive review of P2P energy trading related blockchain technology applications are presented. In addition, an extended (P2P) energy trading multi-layer architecture model is demonstrated where the use of blockchain technology is integrated. Finally, a P2P energy trading case scenario was explained using an Ethereum-based testbed. P2P energy trading allows consumers to become prosumers of electricity in a more efficient, trustable, and profitable way. It is expected that there will be new and improved versions of blockchain and followup technologies which will be released to accelerate the transformation of the decentralized sharing economy in the future. Detailed technology road-mapping, which uses the more advanced version of the multi-layer energy architecture model with corresponding ICT components, and integration of the existing early-stage blockchain testbed to a real-time power system emulator, are two planned extensions of the proposed work which was presented in this article.

\section{Acknowledgements}

There are no acknowledgements for this publication.

\section{References}

[1] Silvente J, Kopanos GM, Pistikopoulos EN, Espuña A. A rolling horizon optimization framework for the simultaneous energy supply and demand planning in microgrids. Applied Energy, 2015; 155: 485-501.

[2] Eltawil MA, Zhao Z. Grid-connected Photovoltaic Power Systems: Technical and Potential Problems-A Review. Renewable Sustainable Energy Reviews, 2010; 14:112-129.

[3] Cali U. Grid and Market Integration of Large-Scale Wind Farms Using Advanced Wind Power Forecasting: Technical and Energy Economic Aspects, kassel university press GmbH, Germany, 2010

[4] Bahrami S, Amini MH. A Decentralized Framework for Real-time Energy Trading in Distribution Networks with Load and Generation Uncertainty, arXiv: 1705.02575, 2017

[5] Nieße A, Lehnhoff S, Tröschel M, Uslar M, Wissing C, Appelrath HJ. Market-based self-organized provision of active power and ancillary services: An agent-based approach for smart distribution grids. In: Complexity in engineering. IEEE; 1-5, 2012.

[6] Duetsch G, Steinecke N. Use cases for blockchain technology in energy \& Commodity Trading, PrivewaterhouseCoopers GmbH Wirtschaftspruefungsgesellschaft, July 2017.

[7] Lo Prete C, \& Hobbs, BF. A cooperative game theoretic analysis of incentives for microgrids in regulated electricity markets. Applied Energy, 2016; 169: 524-541.

[8] Open Utility. (2016). Glimpse into the future of Britain's Energy Economy. [Online]. Available: https://s3-eu-west1.amazonaws.com/ou-publications/piclo-trial-report.pdf

[9] Power L. (2018). Power Ledger Whitepaper. [Online]. Available: https://powerledger.io/media/Power-Ledger-Whitepaperv8.pdf

[10] Lo3. (2018). Transactive Grid Project. [Online]. Available: http://transactivegrid.net

[11] PWC Global Power and Utilities. (2017). Blockchain opportunity for energy producers and consumers. [Online]. Available: https://www.pwc.com/gx/en/industries/assets/pwc-blockchain-opportunity-for-energy-producers-and-consumers.pdf

[12] Condos J, Sorrell WH, Donegan SL. Blockchain technology: opportunities and risks. Research report. State of Vermont, USA, 2016.

[13] Buterin V. (2014). Consensus mechanisms used in blockchain. Available: https://blog. ethereum.org/2014/11/25/proof-stakelearned-love-weak-subjectivity/

[14] Nakamoto, Satoshi. (2009). Bitcoin: A peer-to-peer electronic cash system. [Online]. Available: https://bitcoin.org/bitcoin.pdf

[15] Atzori M. (2016). Blockchain-based architectures for the Internet of things: a survey. Available: https://ssrn.com/abstract=2846810

[16] Buntix JP. (2014). IOTA: Internet of things without the blockchain? Available: http:// bitcoinist.net/iota internet-thingswithout-blockchain

[17] Munsing E, Mather J, and Moura S. Blockchain for decentralized optimization of energy resources in microgird networks. IEEE Conference on Control Technology and Applications (CCTA). USA, 2017. 
[18] Li Z, Kang J, Yu R, Ye D, Deng Q, Zhang Y. Consortium blockchain for secure energy trading in industrial internet of things. IEEE Transactions on Industrial Infiormatics, 2017.

[19] Pop C, Cioara T, Antal M, Anghel I, Salomie I, Bertoncini M. Blockchain Based Decentraalized Management of Demand Response Programs. In: Smart Energy Grids. Sensors, MDPI, 2018.

[20] Mengelkamp E, Gaerttner J, Rock K, Kessler S, Orsini L, Weinhardt C. Designing Microgrid Energy Markets / A Case Study: The Brooklyn Microgirds. Applied Energy, 2018.

[21] Mihaylov M, Jurado S, Avellana N, Van Moffaert K, Abril I.M de, Nowe A. NRGcoin: Virtual Currency for Trading Renewable Energy Smartgrid. In: International Conference on the European Energy Market ( EEM 2014). IEEE, 1-6, 2014.

[22] Chenghua Z, Jianzhong W, Meng C, Yue Z, Chao L. A bidding system for peer-to-peer energy trading in a grid-connected microgrid. Energy Procedia, 2016; 103: 147-152.

[23] CEN-CENELEC-ETSI Smart Grid Coordination Group. (2012). Smart Grid Reference Architecture. [Online]. Available: https://ec.europa.eu/energy/sites/ener/files/documents/xpert_group1_reference_architecture.pdf

[24] Porsinger, Tobias and Janik, Przemyslaw and Leonowicz, Zbigniew and Gono, Radomir. Modelling and optimizing in microgrids. Energies, 10: 523, 2017.

[25] Porsinger T, Janik P, Leonowicz Z, Gono R. Component modelling for microgrids. In: Proc. of the 2016 IEEE $16^{\text {th }}$ Internationa Conference on Environment and Electrical Engineering (EEEIC), June 2016.

[26] Blouin MR, Serrano R. (2001). A decentralized market with common values uncertainty: non-steady states. Rev Econ Stud, 68:323-46, 2001.

[27] Block C, Neumann D, Weinhardt C. A market mechanism for energy allocation in micro-chp grids. In: Proc. the 41st annual Hawaii international conference on system sciences. IEEE; 172-172, 2008.

[28] Lamparter S, Becher S, Fischer JG. An agent-based market platform for smart grids. In: Proceedings of the 9th international conference on autonomous agents and multiagent systems, 2010:1689-96.

[29] Ilic D, Da Silva PG, Karnouskos S, Griesemer M. An energy market for trading electricity in smart grid neighbourhoods. In: 6th IEEE international conference on digital ecosystems technologies (DEST),2012:1-6.

[30] Liu N, Yu X, Wang C, Li C, Ma L and Lei. J. (2017) Energy-Sharing Model With Price-Based Demand Response for Microgrids of Peer-to-Peer Prosumers. In: IEEE Transactions on Power Systems, 3569-3583.

[31] North Carolina Solar Center. (2015). A Residential Customer Guide to Going Solar: Duke Energy Carolina Version. Available: https://nccleantech.ncsu.edu/wp-content/uploads/Duke-Energy-Carolinas-Solar-Guide-FINAL-3.pdf

[32] Ethereum Community. (April 2018). Introduction: What is Ethereum? [Online]. Available: http://ethdocs.org/en/latest/introduction/what-is-ethereum.html

[33] Dotau, S. (April 2018). What is Geth? [Online]. Available: http://www. talkcrypto.org/blog/2018/01/23/what-is-geth/ 